\title{
Comparative Performance and Computational Approach of Humic Acid Removal by Clay Adsorption
}

\author{
Chao Yu ${ }^{1}$, Jiaqian Jiang ${ }^{2 *}$ \\ ${ }^{1}$ Department of Mathematics, East China Normal University, Shanghai, China; ${ }^{2}$ Division of Civil, Chemical and Environmental En- \\ gineering, Faculty of Engineering and Physical Sciences, University of Surrey, Guildford, UK. \\ Email: J.Jiang@surrey.ac.uk
}

Received January $3^{\text {rd }}, 2010$; revised February $3^{\text {rd }}, 2010$; accepted February $3^{\text {rd }}, 2010$.

\begin{abstract}
The effective removal of humic acid is an important factor influencing the quality of treated waters. Adsorption is one of major techniques used for the removal of humic acid. This study demonstrated that modified clays could be used as alternatives to activated carbons for adsorbing humic acid. Both Al-Fe modified and Fe modified clays had high affinity to humic acid and then high removal efficiency. Al-modified clay had less removal capacity for adsorbing humic acid. Mathematics formulas were developed to predict the adsorption performance of modified clays for the humic acid removal via the parameters of $U V_{254}$ absorbance and DOC concentrations. The optimal clay dose could be predicted using the developed model. The F test was used to validate the model developed by examining if it fells into the reject field. The reject field varied according to each $F$ test. The results showed that the model developed was $99 \%$ confident and can be used to perform the simulation.
\end{abstract}

Keywords: Adsorption, Clay, Humic Aid (HA), Mathematics Approach, Modification, Water Treatment

\section{Introduction}

Humic acid in surface water causes a lot of problems such as colour, taste, odour and lower efficiency in water treatment process. In addition to this, in the chlorination process, humic acid reacts with chlorine and produces disinfection-by-products (DBPs) [1]. The World Health Organization (WHO) has recommended the maximum concentrations of the DBPs and these parameters have been regulated in most countries' environmental agencies. The effective removal of humic acid is thus an important factor influencing the quality of treated waters. Among techniques used for the removal of humic acid, coagulation, adsorption and membrane processes are widely adapted.

Clay is one of the most common earth's minerals, which are the residue of weathering or hypothermal action. The classification and origin of clay depends on particle size, physical characteristics, chemical compositions and common crystal structural characteristics. Clay's size is less than 2 micrometers with plastic properties when moist. Fundamentally, clay exhibits a layered structure and itself can be subdivided into groups according to its underlying structure and layer's charge. An ideal structure of the most rigid clays is the 2:1-layered silicates which can be seen in Figure 1. The 2:1 notation means that the host layers consist of two tetrahedral sili- cate sheets sandwiching one octahedral sheet. The two other subclasses of clays have a 1:1 layer type and a 2:1 inverted ribbon structure, respectively [2]. At the central of the tetrahedral layers are silicon or aluminium ions, while the number of $\mathrm{A} 1$ ions in tetrahedral sites determines the net negative charge of the host layer. Those oxygens forming the tetrahedral bases border the interlamellar gallery and are arranged in hexagonal rings that form a kagom'e lattice.

The approximate chemical formula for the verminculites is $\left(\mathrm{Mg}_{3}\left(\mathrm{Si}_{3} \mathrm{Al}\right) \mathrm{O}_{10}(\mathrm{OH})_{2}\right)\left(\mathrm{Mg}_{0.5}\left(\mathrm{H}_{2} \mathrm{O}\right)_{y}\right)$; where the first set of brackets denotes the host layer, the second set denotes the guest layer, and the hydration state is variable. The host layers in clays can adopt a number of interesting stacking arrangements to form ordered, partially ordered, or disordered three-dimensional structures. Particular clays are prone to form poly-types in which different stacking sequences are associated with lateral layerto-layer shifts.

Overall, natural mineral clays possess specific surface chemical properties, e.g., cation exchange capacity, and adsorptive affinity for some organic and inorganic compounds, and then have attracted research interesting to investigate the potential use of clays as adsorbents for treating heavy metals and organic pollutants, or as coagulant aids for improving the settling performance in coagulating low particle content water. By replacing the 


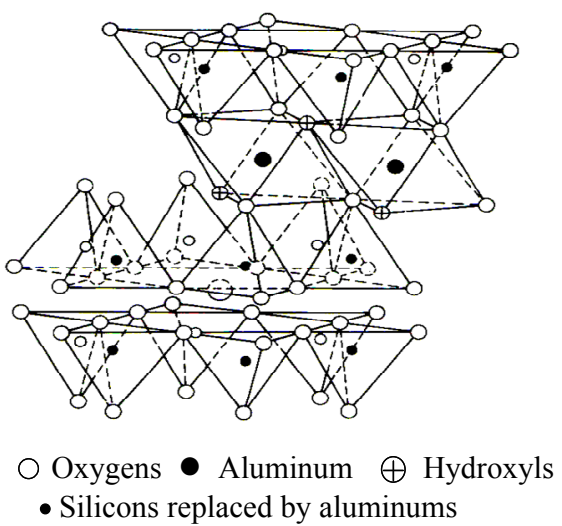

Figure 1. Diagrammatic illustration of 2:1 layer lattice (after [2])

natural inorganic exchange cations with alkylammonium ions, clay surfaces are converted from being primarily hydrophilic to hydrophobic, which enable them to interact strongly with organic vapours and organic compounds dissolved in water [3]. Previous studies [4,5] have demonstrated that the polymeric $\mathrm{Al} / \mathrm{Fe}$ species are the most efficient coagulating/adsorbing chemicals for removing natural and synthetic organic impurities in potable water treatment. The combination of the natural mineral clays with polymeric $\mathrm{Al} / \mathrm{Fe}$ species may produce somewhat optimal properties and enhance the adsorption of metal and organic compounds from the solution. The feasibility of this idea has been confirmed by preceding work [6$10]$, where, modified clays had comparatively great affinities for the heavy metals, and phenol and dye structured pollutants.

The aim of this paper is to use modified clays for humic substances removal and to develop a model to forecast the operating conditions based on the experimental results of using modified clay to adsorb humic acid. It is expected that using the developed model, the most efficient outcome of adsorption of humic acid could be predicted.

\section{Materials and Methods}

\subsection{Modifying and Characterising Clays}

The raw clay used in this study, montmorillonites KSF, and the other chemicals were supplied by Sigma-Aldrich Chemicals Corporation UK. The modification of clays was following an established procedure [7]. The modification involved with the mixing of the given amount of clays with polymeric metal species for four hours at $55^{\circ} \mathrm{C}$ and then the mixtures were separated by filtration to obtain the solid phase of the modified clays. The resulting clays were dried using a freeze dryer (Dry Winner3, HETO Ltd., UK) operating at $-0.5 \mathrm{MPa}$ and $-52^{\circ} \mathrm{C}$. The chemical composition of the modified clays were analysed using X-ray Fluorescence (XRF), and the XRF data was collected on a Philips
PW1480 XRF Spectrometer.

The clays used in the study were raw montmorillonites KSF (termed as raw clay), poly-aluminium modified montmorillonites KSF (Al-clay), poly-iron modified montmorillonites KSF (Fe-clay) and poly-aluminium and iron modified montmorillonites KSF (Al-Fe-clay).

\subsection{Procedures of Adsorption Experiments and Water Quality Measurement}

The model water containing humic acid (HA) was prepared using a commercial HA (Fisher, UK), and the HA concentration was $6.5 \mathrm{mg} / \mathrm{L}$, giving $\mathrm{UV}_{254}$ abs of $151 / \mathrm{m}$ and dissolved organic carbon (DOC) concentration 3.2 $\mathrm{mg} / \mathrm{L}$ as $\mathrm{C}$.

The adsorption experiments were carried out using the batch equilibration technique. For each isotherm, given amount of clay was weighed into $40 \mathrm{~mL}$ polypropylene centrifuge tubes, and $30 \mathrm{~mL}$ of the above stated HA solution were added. The $\mathrm{pH}$ value of HA solution was preadjusted to 5. The suspensions were mixed on a rotary tumbler for 4 hours, which has been tested to be sufficient to reach the equilibrium status under the study conditions. After phase separation by centrifugation, the concentration of HA in the supernatant was determined by UV- absorbance at wavelength of $254 \mathrm{~nm}$ and DOC analysis. The analytical procedures were following the AWWA standard methods [11]. The adsorbed HA quantities were then determined using the mass balance equation:

$$
C_{s} m=V\left(C_{0}-C_{e}\right)
$$

where, $C_{s}$ is sorbed HA concentration on clay $(\mathrm{mg} / \mathrm{g}), m$ is the weight of clay used $(\mathrm{g}), V$ is volume of HA solution (L), $C_{0}$ is HA initial concentration (here expressed as DOC $\mathrm{mg} / \mathrm{L}$ ), and $C_{e}$ is $\mathrm{HA}$ equilibrium concentration (DOC $\mathrm{mg} / \mathrm{L}$ ). Percentage removal of humic acid was calculated based on the original and treated DOC concentrations.

\subsection{Mathematical Approach}

The regression procedures and the least square method were used to set up a model and to analyze the data. In terms of the experimental results of humic acid removed by clays, a model was developed to forecast humic acid removal efficiency by adsorption with clays. Finnaly, the F test was used to validate the model developed.

\section{Results and Discussion}

\subsection{Characterisation of the Modified Clays}

Figure 2 shows an example XRD traces for the modified montmorillonites. The peaks marked by (x) are the $\mathrm{d} 001$ reflections indicative of 2:1 swelling clays. The other peaks are impurities corresponding to quartz, plagioclase feldspar, illite and mica. Illite is a non-swelling 2:1 clay, mica is a non-swelling 2:1 phyllosilicate (sheet silicate) 
mineral and plagioclase is tectosilicate (three dimensional structure similar to zeolite framework).

The XRD results also demonstrated that increase in basal spacing (which is an indication of expanding clay's inter-layers; a high basal spacing value means more inter-layer volume) occurs in the modified montmorillonites but the extents of changes are very different (Table 1). The values of d001 basal spacing of Alor Fe-clays only slightly increased or was different from that of the raw clay but $\mathrm{d} 001$ basal spacing of AlFe-clay increased markedly. The possible reason for this could be that the single Al or Fe polymeric species have probably undergone subsequent hydrolysis prior to XRD analysis, resulting in partial collapse of the interlayer spacing back to near the original value. However, polymeric aluminium-iron species probably are stable, which results in the larger spacing being retained. However, the d001 basal spacing values alone cannot explain the modification mechanism, which is the exchange of the interlayer $\mathrm{Ca}^{2+}$ ions for the polymeric $\mathrm{Al}$ or $\mathrm{Fe}$ species in solution. The XRF analysis revealed that $\mathrm{Ca}^{2+}$ content in the treated clays signifycantly decreased and which is equivalent to $99.5 \%$ for the Al- or Fe-clays, and $98.6 \%$ for the Al-Fe-clay, indicating that the polymeric $\mathrm{Fe}$ or Al species are definitely entering the internal structure of the clays.

\subsection{Adsorption of Humic Acid}

Tables 2 and $\mathbf{3}$ show the adsorption of humic acid vs. doses of clays. Al-Fe- and Fe-clays demonstrated superior

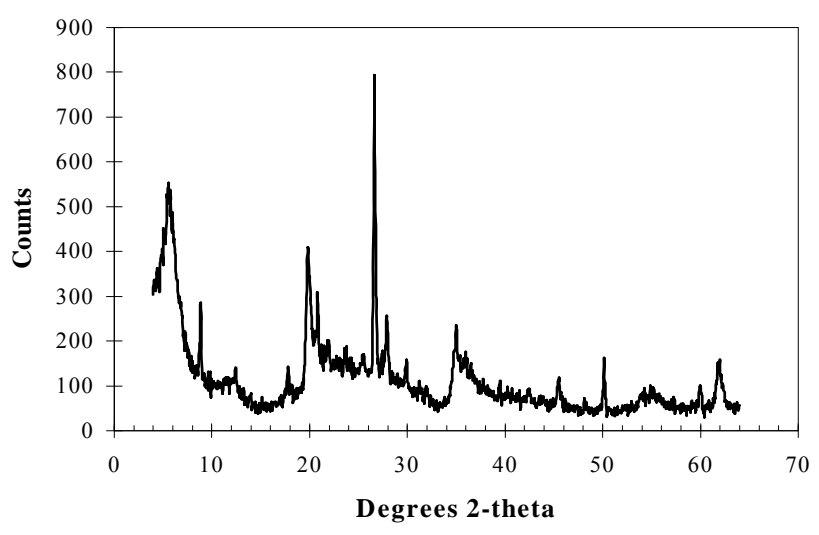

Figure 2. XRD trace of $\mathrm{Al}_{13}$-polycation modified montmorillonite

Table 1. Basal spacing for modified montmorillonites KSF clays

\begin{tabular}{cc}
\hline Type of clay & Basal spacing $(\mathrm{d} 001) / \AA$ \\
\hline Raw clay & 15.5 \\
Al-clay & 15.3 \\
Fe-clay & 15.9 \\
Al-Fe clay & 17.8 \\
\hline
\end{tabular}

humic acid removal efficiency when doses were above $400 \mathrm{mg} / \mathrm{L}$. whilst Al-clay did not show good adsorption performance under study conditions. It is well documented that the interaction of iron (III) with humic acid involves complexing, charge neutralization, precipitation and adsorption [12]. Most common $\mathrm{pH}$ used for removing humic acid is 4-6 and complexing species is $\mathrm{Fe}(\mathrm{III})$, therefore, both Al-Fe-clay and Fe- clay performed superior to Al-clay. The best performance was achieved by Al-Fe clay since the modifier used in this clay is polymeric alumino-iron species, which has been demonstrated to have the highest cationic charge in comparison with other Al/Fe metal species [13].

The superior adsorption performance of modified clays could be attributed to their specific properties; i.e., high hydrophobicity and specific chemical complexation. After modification, the modified clays either became more hydrophobic in nature or increased interactions with functional groups of the humic acid (e.g., carboxyl, hydroxyl and carbonyl). Most possibly, the combination of two mentioned mechanisms can be used to explain the enhanced humic acid adsorption with Al-Fe-clays.

\subsection{Development of Mathematics Models}

The following formula (Equation 1) was set up to be fitted with the adsorption operating conditions stated pre-

Table 2. DOC adsorption vs. clay dose

\begin{tabular}{cccc}
\hline $\begin{array}{c}\text { Clay dose } \\
(\mathrm{mg} / \mathrm{L})\end{array}$ & Al-Fe- & Al- & Fe- \\
\hline 0 & 3.1 & 3.2 & 3.2 \\
200 & 2.85 & 2.86 & 3.47 \\
400 & 0.16 & 2.44 & 0.16 \\
600 & 0.09 & 2.30 & 0.09 \\
800 & 0.08 & 2.11 & 0.09 \\
1000 & 0.09 & 1.93 & 0.10 \\
\hline
\end{tabular}

Table 3. $\mathrm{UV}_{254}$ adsorption vs. clay dose

\begin{tabular}{cccc}
\hline $\begin{array}{c}\text { Clay dose } \\
(\mathrm{mg} / \mathrm{L})\end{array}$ & $\mathrm{Al}-\mathrm{Fe}-$ & $\mathrm{Al}-$ & $\mathrm{Fe}-$ \\
\hline 0 & 0.252 & 0.257 & 0.260 \\
200 & 0.232 & 0.230 & 0.282 \\
400 & 0.013 & 0.196 & 0.013 \\
600 & 0.007 & 0.185 & 0.007 \\
800 & 0.006 & 0.169 & 0.007 \\
1000 & 0.007 & 0.155 & 0.008 \\
\hline
\end{tabular}


viously, i.e., that the $\mathrm{Al}$ and $\mathrm{Fe}$ have an equally fixed ratio towards clay when they were modified, $\mathrm{Al}, \mathrm{Fe}$ and clay are independent variables, i.e. they won't interfere with each other when performing as adsorbents.

$$
y=\beta_{0}+\beta_{1} x_{1}+\beta_{2} x_{2}+\beta_{3} x_{3}+\varepsilon
$$

where y represents the remaining DOC or $\mathrm{UV}_{254}$ treated by Al-Fe-clay; $\mathrm{x} 1, \mathrm{x} 2$, and $\mathrm{x} 3$ represent the remaining DOC or $\mathrm{UV}_{254}$ treated by Al-, Fe- and raw clay respectively; $\beta_{i}$ are unknown parameters $(\mathrm{i}=0,1,2,3) ; \varepsilon$ is the random error item which satisfy:

$$
E(\varepsilon)=0 \text { and } \operatorname{Var}(\varepsilon)=\sigma^{2}
$$

The purpose of the above model is to predict the adsorption performance of either Al-Fe-clay $(y)$ or other clays $\left(x_{i}\right)$. The influence of three factors were considered: $\mathrm{Al}$ content, Fe content and raw clay. And the least square method was used to obtain $\beta_{i}$. Take the form

$$
y_{i}=\beta_{0}+\beta_{1} x_{i 1}+\beta_{2} x_{i 2}+\beta_{3} x_{i 3}+\varepsilon_{i}, i=1,2,3 \ldots, n
$$

where, $n \geq 3$.

A matrix form was written as follows:

$$
\begin{gathered}
Y=\left(y_{1}, y_{2}, \ldots, y_{n}\right)^{T} \\
X=\left(\begin{array}{cccc}
1 & x_{11} & x_{12} & x_{13} \\
\vdots & \vdots & \ddots & \vdots \\
1 & x_{n 1} & x_{n 2} & x_{n 3}
\end{array}\right)_{n \times 4} \\
\beta=\left(\beta_{0}, \beta_{1}, \beta_{2}, \beta_{3}\right)^{T} \\
\varepsilon=\left(\varepsilon_{1}, \varepsilon_{2}, \ldots, \varepsilon_{n}\right)^{T}
\end{gathered}
$$

Thus, the original equation was written as:

$$
Y=X \beta+\varepsilon
$$

Compute the parameters. According to the least square method, the estimated vector $\hat{\beta}$ was found which should satisfy:

$$
\begin{aligned}
& Q\left(\hat{\beta}_{0}, \hat{\beta}_{1}, \hat{\beta}_{2}\right)=\sum_{i=1}^{n}\left(y_{i}-\hat{\beta}_{0}-\hat{\beta}_{1} x_{i 1}-\hat{\beta}_{2} x_{i 2}-\hat{\beta}_{3} x_{i 3}\right)^{2} \\
& =\min _{\beta_{0}, \beta_{1}, \beta_{2}} \sum_{i=1}^{n}\left(y_{i}-\beta_{0}-\beta_{1} x_{i 1}-\beta_{2} x_{i 2}-\beta_{3} x_{i 3}\right)^{2}
\end{aligned}
$$

The parameters were then computed by using method from multivariable calculus and were written in the matrix form:

$$
X^{T}(Y-X \hat{\beta})=0
$$

Since $X^{T} X$ is a non-singular matrix, thus the formula was obtained to compute $\beta$

$$
\hat{\beta}=\left(X^{T} X\right)^{-1} X^{T} Y
$$

In real practice, Matlab could be used to compute the above calculation.

For the DOC value,

$\hat{\beta}=(-0.1813,0.0833,0.8057,0.1391)^{\mathrm{T}}$, and then Equation 2 was obtained,

$$
\hat{y}_{D O C}=-0.1813+0.0833 x_{1}+0.8075 x_{2}+0.1391 x_{3}
$$

Similar procedures were applied to $\mathrm{UV}_{254}$-abs removal prediction, or, Equation 3:

$$
\hat{y}_{U V}=-0.013+0.073 x_{1}+0.8072 x_{2}+0.1448 x_{3}
$$

Figures 3 and $\mathbf{4}$ show the remaining DOC concentrations and $\mathrm{UV}_{254}$ abs. after clay adsorption via either the experimental and the model simulated data. Both approaches deliver significantly consistent results indicating that the developed model could be used to predict the humic acid adsorption performance by clays.

\subsection{Hypothesis Test}

The $\mathrm{F}$ test was used to validate the model developed, which determines whether $\mathrm{y}$ and $\mathrm{x}_{1}, \mathrm{x}_{2}$ and $\mathrm{x}_{3}$ are linear correlated at a significant level (alpha equals to 0.01), i.e., whether it is appropriate to represent $\mathrm{y}$ with beta $_{0}+$

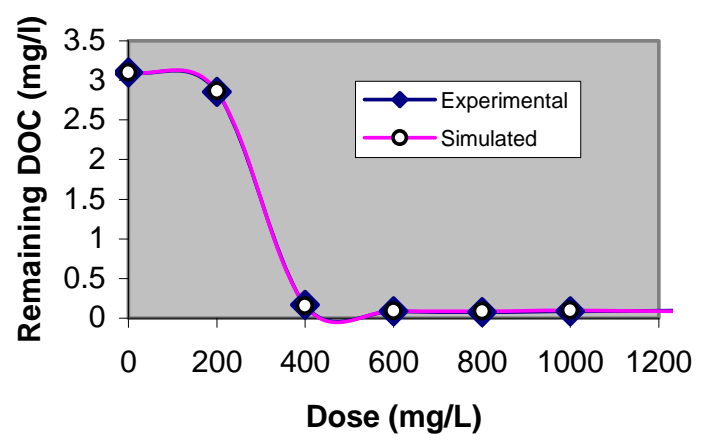

Figure 3. Model simulated and experimental remaining DOC

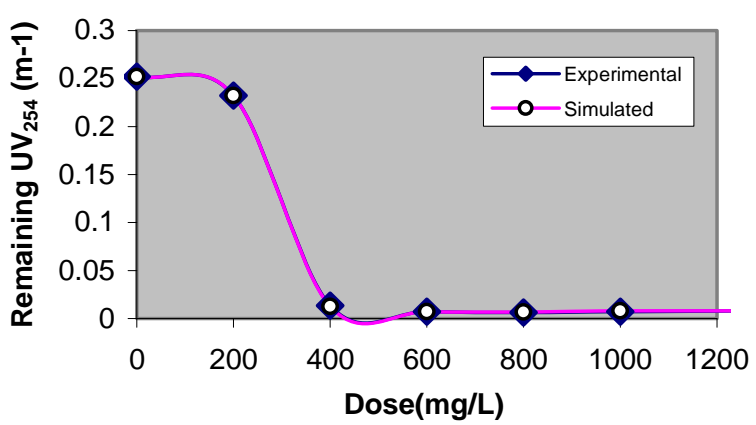

Figure 4. Model simulated and experimental remaining $\mathbf{U V}_{254}$ 
beta $_{1} * \mathrm{x}_{1}+$ beta $_{2} * \mathrm{x}_{2}+$ beta $_{3} * \mathrm{x}_{3}$ with the confidence level of $99 \%$. The $\mathrm{F}$ value is examined to see if it fells into the reject field. If it is, the developed model is wrong, and has to be reconstructed. The reject field varies according to each $\mathrm{F}$ test.

As shown in the following equations, that the $\mathrm{F}$ test needs three components to locate, which are $0.01,4$ and 3 , respectively. The 0.01 means the confident level (equals to 99\%), 4 and 3 are the degree of freedom which are set due to the data numbers. 16.69 stands for the reject field (obtainable from the table provided with statistics books). If the $\mathrm{F}$ value computed is less than 16.69 , then the hypothesis that original model is correct would be rejected. As the $\mathrm{F}$ value in this case is greater than 16.69 (Equations 4 and 5), it means that the developed model was $99 \%$ in confidence (with the coefficients beta - beta $_{3}$ are not all zero) for its accuracy, and can be used to simulate and predict the adsorption performance.

$$
\begin{gathered}
H_{0}: \beta_{0}=\beta_{1}=\beta_{2}=\beta_{3}=0 \text { Versus } \\
H_{1}:\left(\beta_{0}, \beta_{1}, \beta_{2}, \beta_{3}\right) \neq(0,0,0,0) \\
S_{T}=l_{y y}=\sum_{i=1}^{n}\left(y_{i}-\bar{y}\right)^{2}=\sum_{i=1}^{n}\left(\hat{y}_{i}-\bar{y}\right)^{2}+\sum_{i=1}^{n}\left(y_{i}-\hat{y}_{i}\right)^{2} \\
=S_{R}+S_{E} \\
F=\frac{\frac{S_{R}}{4}}{\frac{S_{E}}{n-4-1}} \sim F(4, n-4-1) \\
=26322.29>F_{0.01}(4,3)=16.69 \\
F_{U V_{254}} \\
F_{D O C}=7748.875>F_{0.01}(4,3)=16.69
\end{gathered}
$$

So $\mathrm{H}_{0}$, was rejected and the parameters can be fit to the model significantly at the $99 \%$ confidence.

The developed models were verified, and then they can be used to predict that when the clays' doses are about $400 \sim 600 \mathrm{mg} \mathrm{l}^{-1}$, the overall adsorption of $\mathrm{UV}_{254}$ and DOC could reach to the maximum. Furthermore, if the remaining $\mathrm{UV}_{254}$ or $\mathrm{DOC}$ could be known, the outcome of Al-Fe modified clay at the same dose level could be forecasted.

\section{Conclusions}

This study demonstrated that modified clays could be used as alternatives to activated carbons for humic acid removal. Both Al-Fe modified and Fe modified clays have high affinity to humic acid and then high removal efficiency. Al-modified clay has less removal capacity for adsorbing humic acid. Higher d-spacing values of Al-Fe modified and Fe modified clays could explain such phenomena.

Mathematics formulas were developed to predict the adsorption performance of modified clays for the humic acid removal via the parameters of UV254 absorbance and DOC concentrations. The optimal clay dose could be predicted using the developed models.

The F test was used to validate the model developed by examining if it fells into the reject field. The reject field varied according to each $\mathrm{F}$ test. The results showed that the model developed was $99 \%$ confident and can be used to perform the simulation.

\section{Acknowledgement}

The authors would like to thank UK Engineering Physical Science Research Council (EPSRC) for funding Chao Yu's internship at the University of Surrey under the Knowledge Transfer Award Scheme.

\section{REFERENCES}

[1] B. M. Chow and P. V. Robert, "Halogenated by products formation by $\mathrm{ClO}_{2}$ and $\mathrm{Cl}_{2}$," Journal of the Environmental Engineering Division, ASCE, Vol. 107, No. 4, pp. 609 $615,1981$.

[2] G. W. Brindley and G. Brown, "Crystal structures of clay minerals and their X ray identification," Mineralogical Society, London, pp. 495, 1980.

[3] H. T. Zhao and G. F. Vance, "Sorption of trichloroethylene by organo-clays in the presence of humic substances," Water Research, Vol. 32, No. 12, pp. 3710-3716, 1998.

[4] J. Q. Jiang and N. J. D. Graham, "Enhanced coagulation using $\mathrm{Al} / \mathrm{Fe}$ (III) coagulants: Effect of coagulant chemistry on the removal of colour-causing NOM," Enviromental Technology, Vol. 17, No. 9, pp. 937-950, 1996.

[5] J. Q. Jiang and N. J. D. Graham, "Preparation and characterization of an optimal polyferric sulphate (PFS) as a coagulant for water treatment," Journal of Chemical Technology \& Biotechnology, Vol. 73, pp. 351-358, 1998.

[6] J. Q. Jiang, C. Cooper, and S. Ouki, "Comparison of modified montmorillonite adsorbents, Part I: Preparation, characterization and phenol adsorption," Chemosphere, Vol. 47, No. 7, pp. 711-716, 2002.

[7] J. Q. Jiang and Z. Zeng, "Comparison of modified montmorillonite adsorbents, Part II: The effects of the type of raw clays and modification conditions on the surface properties and adsorption performance of modified clays," Chemosphere, Vol. 53, No. 1, pp. 53-62, 2003.

[8] J. Q. Jiang, Z. Q. Zeng, and P. Pearce, "Preparation and use of modified clay coagulants for wastewater treatment," Water, Air, \& Soil Pollution, Vol. 158, No. 1, pp. 53-65, 2004.

[9] S. Richards and A. Bouazza, "Phenol adsorption in organo-modified basaltic clay and bentonite," Applied Clay Science, Vol. 37, No. 1-2, pp. 133-142, 2007.

[10] E. I. Unuabonah, K. O. Adebowale, and F. A. Dawodu, "Equilibrium, kinetic and sorber design studies on the adsorption of Aniline blue dye by sodium tetraboratemodified Kaolinite clay adsorbent," Journal of Hazardous Materials, Vol. 157, No. 2-3, pp. 397-409, 2008.

[11] APHA, AWWA, and WEF, "Standard Methods for the 
examination of water and wastewater," 18th Edition, American Publication Health Association, Washington, DC, 1992.

[12] C. P. Huang and H. L. Shiu, "Interactions between alum and organics in coagulation," Colloids and Surfaces A: Physicochemical and Engineering Aspects, Vol. 113, No.
1-2, pp. 155-163, 1996.

[13] J. Q. Jiang, "Development of coagulation theory and pre-polymerised coagulants for water treatment," Separation and Purification Methods, Vol. 30, No. 1, pp. 127 142, 2001. 